OPEN ACCESS

Edited by:

Yanting $W u$,

Fudan University, Shanghai, China

Reviewed by:

Wanzhu Jin,

Chinese Academy of Sciences (CAS),

China

Guannan Zhou,

Fudan University, China

${ }^{*}$ Correspondence:

Xiaoying Ding

xiaoyingding@126.com

Chenhong Zhang

zhangchenhong@sjtu.edu.cn

${ }^{\dagger}$ These authors have contributed equally to this work

Specialty section: This article was submitted to

Reproduction,

a section of the journal

Frontiers in Endocrinology

Received: 31 October 2021 Accepted: 31 December 2021

Published: 02 February 2022

Citation:

Wang $X, X u T$, Liu R, Wu G, Gu L, Zhang $Y$, Zhang F, Fu H, Ling $Y$, Wei $X$,

Luo $Y$, Shen J, Zhao L, Peng $Y$, Zhang $C$ and Ding $X$ (2022) High-Fiber Diet or Combined With Acarbose Alleviates Heterogeneous Phenotypes of Polycystic Ovary Syndrome by

Regulating Gut Microbiota.

Front. Endocrinol. 12:806331. doi: 10.3389/fendo.2021.806331

\section{High-Fiber Diet or Combined With Acarbose Alleviates Heterogeneous Phenotypes of Polycystic Ovary Syndrome by Regulating Gut Microbiota}

Xuejiao Wang ${ }^{1 \dagger}$, Ting $\mathrm{Xu}^{2+}$, Rui Liu ${ }^{2}$, Guojun $\mathrm{Wu}^{3}$, Liping Gu ${ }^{1}$, Yahui Zhang ${ }^{1}$, Feng Zhang ${ }^{2}$, Huaqing $\mathrm{Fu}^{2}{ }^{2}$, Yunxia Ling ${ }^{1}$, Xiaohui Wei ${ }^{1}$, Yunchen Luo ${ }^{1}$, Jian Shen ${ }^{4}$, Liping Zhao ${ }^{2,3}$, Yongde Peng ${ }^{1}$, Chenhong Zhang ${ }^{2 *}$ and Xiaoying Ding ${ }^{1 *}$

\begin{abstract}
${ }^{1}$ Department of Endocrinology and Metabolism, Shanghai General Hospital, Shanghai Jiao Tong University School of Medicine, Shanghai, China, ${ }^{2}$ State Key Laboratory of Microbial Metabolism and Ministry of Education Key Laboratory of Systems Biomedicine, School of Life Sciences and Biotechnology, Shanghai Jiao Tong University, Shanghai, China, ${ }^{3}$ Department of Biochemistry and Microbiology and New Jersey Institute for Food, Nutrition, and Health, School of Environmental and Biological Sciences, Rutgers University, New Brunswick, NJ, United States, ${ }^{4}$ Shanghai Centre for Systems Biomedicine, Shanghai Jiao Tong University, Shanghai, China
\end{abstract}

Objective: Gut microbial dysbiosis is associated with high heterogeneity of polycystic ovary syndrome (PCOS); however, studies about gut microbiota targeted clinical intervention in PCOS are limited. Our study aimed to evaluate the effects of high-fiber diet or combined with acarbose on the clinical phenotypes of PCOS, focusing on the possible influence of gut microbiota in this process.

Methods: Twenty-five patients with PCOS were recruited and randomly divided into two groups, W group $(n=14)$ received the WTP diet (a high-fiber diet composed of whole grains, traditional Chinese medicinal foods, and prebiotics), and A group ( $n=11$ ) received the WTP diet combined with acarbose. The follow-up time was 12 weeks. The sex hormonal and glycolipid metabolic parameters, inflammatory factors, brain-gut peptides, and alteration of gut microbiota were evaluated.

Results: The PCOS clinical phenotypes, inflammatory state, and brain-gut peptides secretion were all alleviated in both groups, while the hyperandrogenism, insulin resistance, and brain-gut peptides secretion were better improved in the A group. Alpha and beta diversities were altered more significantly in the A group. Amplicon sequence variants (ASVs) were clustered into 14 co-abundant groups (CAGs) as potential functional groups that may respond to the intervention. The CAGs predominantly comprised of Bifidobacterium and Lactobacillus were more enriched, while the CAGs predominantly comprised of Bacteroides vulgatus, Alistipes, Blautia, Lachnospira, and Roseburia were more inhibited in the A group than in W group. Moreover, the CAGs enriched in the A group had a stronger negative correlation with the luteinizing hormone $(\mathrm{LH}) /$ follicle-stimulating hormone $(\mathrm{FSH})$ ratio, testosterone, homeostasis model 
assessment-insulin resistance (HOMA-IR), $\alpha$-1-acid glycoprotein ( $\alpha$-AGP), and leptin, and positive correlation with adiponectin and spexin, while the CAGs inhibited showed an opposite trend.

Conclusions: High-fiber diet could alleviate the chronic metabolic inflammation, reproductive function, and brain-gut peptides secretion of patients with PCOS, and high-fiber diet combined with acarbose could better improve the PCOS clinical phenotypes. The remodeling of gut microbiota by our intervention may play an important role in these improvements.

Clinical Trial Registration: http://www.chictr.org.cn/showproj.aspx?proj=4500, ChiCTR-TRC-14005075

Keywords: polycystic ovary syndrome, gut microbiota, clinical phenotype, dysbiosis, high-fiber diet, acarbose

\section{INTRODUCTION}

Polycystic ovary syndrome (PCOS) is a common heterogeneous endocrine-metabolic disorder in women of reproductive age. According to the 2003 Rotterdam criteria (1), PCOS is defined by the presence of the two or more of clinical manifestations: clinical or biochemical hyperandrogenism, oligo- or anovulation, and polycystic ovaries. Furthermore, PCOS often coexists with abnormal body fat distribution, insulin resistance, glycolipid metabolism disorders, and pituitary-gonad axis anomaly (2). Hyperandrogenism and insulin resistance, the two core pathogenic factors of PCOS, are positively correlated with each other (3), which suggests that PCOS exists with multiple endocrine axis dysfunctions.

European and American studies show that the consumption of cheese and high-glycemic index starchy sweets is higher and the intake of complex carbohydrates and dietary fibers is lower in patients with PCOS compared to healthy individuals (4-6). Dietary fibers intake of patients with PCOS is negatively correlated with insulin resistance, fasting insulin (FINS), glucose tolerance, and androgen levels (7). The above studies suggest that unreasonable dietary structure may result in obesity and metabolic dysfunction in patients with PCOS. Nevertheless, the mechanisms by which dysbiosis of gut microbiota can be the important trigger points of PCOS heterogeneous endocrine disorder remain largely unexplored.

A poor diet may cause the dysbiosis of gut microbiota homeostasis, termed "gut microbial dysbiosis," and lead to obesity-related diseases $(8,9)$. Human studies show that patients with PCOS have gut microbial dysbiosis compared with healthy controls, and the gut microbial dysbiosis is associated with clinical parameters, including body mass index (BMI), insulin resistance, and testosterone (10-12). Furthermore, gut microbial dysbiosis may be involved in the pathogenesis of PCOS through the gut-brain axis and inflammatory reaction of host $(10,13,14)$. Nowadays, there is a growing interest to use probiotics in patients with PCOS. Two studies show that the probiotics supplementation with Lactobacillus and Bifidobacterium have beneficial effects on reproductive endocrine (15) and metabolic disorders (16), respectively.

Nutritional intervention rich in dietary fibers has been gradually recognized and applied to the improvement of metabolic syndromes such as obesity and type 2 diabetes mellitus (T2DM) by selectively promoting probiotics $(17,18)$. Lots of studies suggest that patients with PCOS have low intake of dietary fibers $(7,19)$. However, studies of the dietary fiber intervention in patients with PCOS are limited. Acarbose, an $\alpha$ glucosidase inhibitor, could inhibit the absorption of starch in the small intestine and lead to starch featuring slowly digested "lente" carbohydrate and with increased delivery of starch into the large intestine eventually (20). Acarbose has been confirmed to increase the gut content of Bifidobacteria (21) and was once used to treat PCOS (22). We speculate that nutritional intervention with a high-fiber diet may be a feasible treatment for PCOS by remodeling the gut microbiota, and acarbose may enhance this remodeling by increasing the delivery of starch into the large intestine.

Up to now, studies on gut-microbiota-targeted intervention of PCOS are limited. This study aimed to evaluate the effects of intervention rich in dietary fibers or combined with acarbose on the clinical phenotypes of patients with PCOS and explore the possible influence of gut microbiota in this process.

\section{MATERIALS AND METHODS}

\section{Participants}

Participants diagnosed with PCOS were recruited from the Department of Endocrinology and Metabolism and Department of Gynecology of Shanghai General Hospital (Shanghai, China). In our study, each participant met all of the three clinical manifestations based on the 2003 Rotterdam criteria, including clinical and/or biochemical hyperandrogenism, oligo- or anovulation, and polycystic ovaries (1). Exclusion criteria included the following: androgen-secreting tumors, adrenal disorders, Cushing's syndrome, hypertension, smoking, drinking, and pregnancy. None of the participants had received 
treatment with hormone drugs, insulin sensitizers, anti-obesity drugs, or antibiotics within 3 months prior to enrollment.

\section{Study Approval}

The Human Research Ethics Committee of Shanghai General Hospital (No. 2014KY091) had approved the study protocol ahead of the enrollment procedure. This clinical trial was registered in Chinese Clinical Trial Registry (ChiCTR-TRC14005075). All participants provided written informed consent.

\section{Study Design}

This study was an open-label, randomized controlled trial. Twenty-five participants (aged 15-41 years) were randomly assigned into two groups (Figure 1). W group $(\mathrm{n}=14)$ received a high-fiber diet composed of whole grains, traditional Chinese medicinal foods, and prebiotics (the WTP diet) for 12 weeks. A group $(n=11)$ received the WTP diet combined with acarbose for 12 weeks. The WTP diet included two ready-toconsume prepared foods [canned gruel and prebiotic powder; Perfect (China) Co., Zhongshan, China]. More specifically, canned gruel was a precooked mixture consisting of 11 wholegrains and traditional Chinese medicinal food that are rich in dietary fibers, including adlay, oat, white hyacinth bean, buckwheat, yam, soybean, red phaseolus bean, peanut, wolfberry, corn, and lotus seed. This canned gruel was $360 \mathrm{~g}$ wet weight per can prepared by the food manufacturer. Prebiotic powder was a powder preparation for infusion (20 g per bag) containing soluble dietary fibers, including resistant dextrin, maltose oligosaccharide, inulin, and fructo-oligosaccharide. All participants were instructed by a dietitian to consume at least $240 \mathrm{~g}$ canned gruel and $60 \mathrm{~g}$ prebiotic powder together with at least of $500 \mathrm{~g}$ fresh vegetables and with adequate amounts of meat, eggs, mushrooms, soy products fruits, and nuts per day. The detailed composition of canned gruel and prebiotic powder is shown in Supplementary Table S2. Participants of the A group were prescribed with acarbose (50 mg; 3 times/day) as a medication. The doses of acarbose (Glucobay, Bayer AG) were unchanged throughout the intervention. All participants received a nutrition education and physical examination at baseline.

\section{Outcomes}

This study was conducted to explore the effects of intervention on the clinical phenotypes of PCOS and the role of gut microbiota in this process. The primary endpoints included the sex hormonal and glycolipid metabolic parameters. Gut microbiota may contribute to the development of PCOS by influencing chronic inflammation of host and gut-brain axis (23). Thus, the inflammatory factors and brain-gut peptides were also evaluated.

Food intake based on the 24-h dietary review questionnaire, anthropometric measurements, and blood and fecal samples were taken every 4 weeks (week $0,4,8$, and 12) during the clinical intervention and follow-up. Blood samples were tested for the sex hormonal and glycolipid metabolic parameters, inflammatory factors, and brain-gut peptides. Fecal samples were used for DNA extraction and 16S rRNA gene sequencing. Dual-energy X-ray absorptiometry (DEXA) was used to measure the body composition. The Ferriman-Gallway hirsutism score was obtained at baseline and week 12 .

\section{Laboratory Measurement}

The standard 75-g oral glucose tolerance test (OGTT) was performed in all participants after an overnight fasting for at least $8 \mathrm{~h}$, and blood samples were collected at the following time point $(0,30,60$, and $120 \mathrm{~min})$ and stored at $-80^{\circ} \mathrm{C}$ until analysis. Biochemical parameters were measured using an automatic biochemical analyzer (AU5800 Clinical Chemistry System, Beckman Coulter, CA, USA). Serum insulin and sex hormones were measured using an automated immunoassay system (Tosoh Bioscience AIA ${ }^{\mathrm{TM}}$ - 1800 Systems, TOSOH Corporation, Tokyo, Japan). HbA1c level was measured using a high-performance liquid chromatography (Bio-Rad Variant II Turbo, Bio-Rad Laboratories, München, Germany). For cytokines and brain-

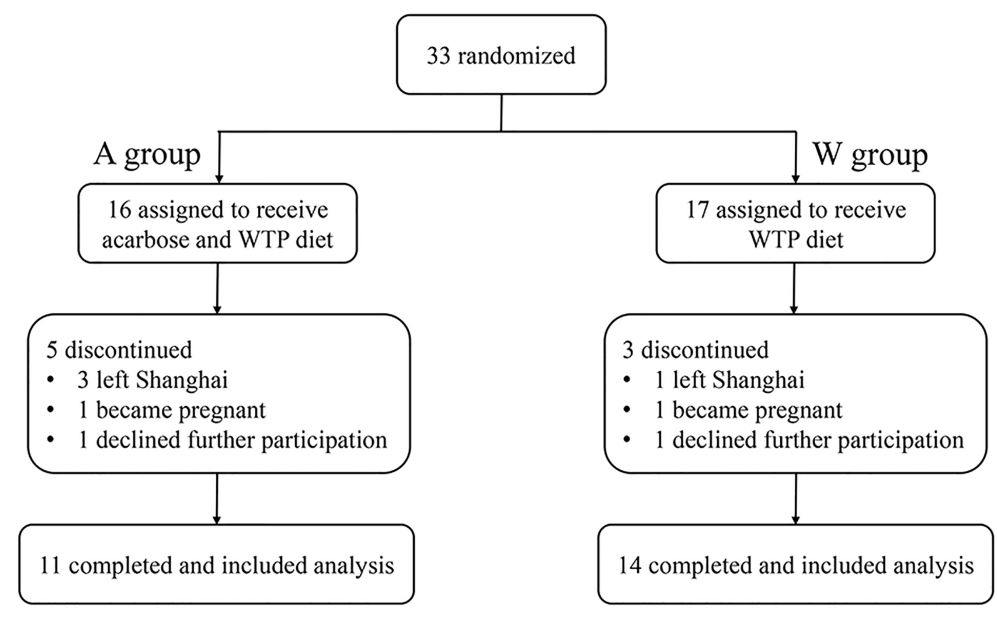

FIGURE 1 | Design and participant flow of clinical trial. 
gut peptides, blood samples were collected according to the kit instructions. Enzyme-linked immunosorbent assays (ELISAs) were used to quantify the level of serum lipopolysaccharide (LPS)-binding protein (LBP) (Hycult Biotech, PA, USA), $\alpha-1$ acid glycoprotein ( $\alpha$-AGP) (Assaypro Inc, MO, USA), leptin and adiponectin (DL develop, Wuhan, China), and orexin and spexin (Phoenix Pharmaceuticals Inc., Burlingame, USA). The intraand inter-assay coefficients of variation were $<5 \%$ and $<10 \%$, respectively.

\section{DNA Extraction and Sequencing}

DNA was extracted from frozen fecal samples as we previously described (24). Forty-four samples from 11 participants in the A group (weeks $0,4,8$, and 12) and 56 samples from 14 participants in the $\mathrm{W}$ group (weeks $0,4,8$, and 12) were sequenced on the Illumina Miseq platform (Illumina, Inc., USA) with Miseq reagent kit v3 (600 cycles) (MS-102-3033, Illumina, USA). Sequencing library of the V3-V4 regions of the 16S rRNA gene was constructed based on a modified version of instruction provided by the manufacturer (Part no. 15044223 Rev. B, Illumina, USA) (25).

\section{Statistical Analysis Clinical Data Statistical Analysis}

Normal distribution of the data was calculated with the Kolmogorov-Smirnov test using SPSS statistical software 19. Variables that were not normally distributed were logtransformed before analysis. Data were expressed as means \pm standard error of mean (SEM) unless otherwise stated. For the clinical and laboratory variables, one-way repeated-measures analysis of variance (ANOVA) with Dunnett's post-hoc test (two tailed) was used for intra-group comparison of different time points (weeks $0,4,8$, and 12), a paired t-test (two tailed) was used for intra-group comparison of week 12 with baseline, and a Mann-Whitney test was used to analyze differences between the $\mathrm{A}$ and $\mathrm{W}$ group at the same time point. A $p$-value $<0.05$ was considered to be statistically significant, ${ }^{*} p<0.05,{ }^{* *} p<0.01$ and ${ }^{* * *} p<0.001$. These statistical analyses were performed using GraphPad Prism software 6.

\section{Bioinformatics and Statistical Analysis}

The 16S rRNA gene sequence data were processed and analyzed on the QIIME2 software (v2018.11) (26). The raw sequence data was demultiplexed and then denoised with DADA2 pipeline ( $\mathrm{q} 2-$ dada2 plugin) (27) to obtain the amplicon sequence variants (ASVs) frequency data table. Alpha diversity metrics (observed ASVs and Shannon index), beta diversity metric (Bray-Curtis and UniFrac distance), and principal coordinate analysis (PCoA) were performed using the q2-diversity after rarefying the samples to 11,000 sequences per sample. Taxonomic assignment for ASVs was performed via the q2-feature classifier (28) using the SILVA rRNA gene database (29). The alpha diversity indexes were compared using the analysis of ANOVA or Mann-Whitney test as the clinical indexes. The intervention-induced structural shifts of gut microbiota were evaluated using Bray-Curtis and weighted UniFrac distances, visualized by PCoA plot using GraphPad Prism software 6, and assessed by the Permutation Multivariate Analysis of Variance (PERMANOVA) analysis using R “vegan” package with 9,999 permutations.

ASVs shared by at least $20 \%$ of all samples were considered as prevalent ASVs. The correlation coefficients between the ASVs were calculated by SparCC algorithm (30). The correlations were converted to a correlation distance ( 1 - correlation coefficients) and then clustered into 14 co-abundant groups (CAGs) using the Ward clustering and PERMANOVA with 9,999 permutations. The ASVs were clustered using the Ward clustering algorithm via MATLAB, and then, the CAG network was visualized in Cytoscape. Wilcoxon matched-pairs signed-rank test was used to compare the relative abundance of CAGs on weeks $4,8,12$ vs baseline in the same group. A Mann-Whitney test was used to compare differences between the $\mathrm{A}$ and $\mathrm{W}$ group at the same time point. $p<0.05$ was considered as differential variable. Microbiome Multivariable Association with Linear Models 2 (MaAslin2) was used to calculate the multivariable associations via generalized linear regression between CAGs and clinical parameters with subjects as random effect and adjustment of age.

\section{RESULTS}

\section{Changes in Macronutrient and Dietary Fibers Intake in Patients with PCOS}

At baseline, there was no significant difference in daily energy and macronutrient intakes between the two groups (Table 1). Both of the two groups were given the WTP diet with a large amount of diverse dietary fibers to perturb the gut ecosystem of

TABLE 1 | Daily energy and macronutrient intake of PCOS before and during the intervention.

\begin{tabular}{|c|c|c|c|c|}
\hline \multirow[t]{2}{*}{ Daily intake } & \multicolumn{2}{|c|}{ A group $(n=11)$} & \multicolumn{2}{|c|}{$W$ group $(n=14)$} \\
\hline & Week 0 & Week 12 & Week 0 & Week 12 \\
\hline Energy, kcal & $1,716.24 \pm 769.21$ & $1,380.63 \pm 240.76$ & $1,624.82 \pm 480.78$ & $1,484.82 \pm 301.58$ \\
\hline Fat, $\mathrm{g}$ & $57.5 \pm 29.33$ & $39.48 \pm 8.14$ & $59.73 \pm 20.43$ & $45.46 \pm 8.98^{\star}$ \\
\hline Protein, g & $71.32 \pm 39.08$ & $50.6 \pm 17.39$ & $66.3 \pm 20.35$ & $58.11 \pm 18.92$ \\
\hline Carbohydrate, g & $243.44 \pm 102.76$ & $241.4 \pm 37.01$ & $219.86 \pm 80.26$ & $243.55 \pm 51.19$ \\
\hline Fiber, $g$ & $15.07 \pm 7.8$ & $51.93 \pm 7.7^{\star \star \star}$ & $14.35 \pm 9.74$ & $48.27 \pm 8.48^{\star \star \star}$ \\
\hline Insoluble fiber, $\mathrm{g}$ & $9.36 \pm 4.85$ & $26.87 \pm 5.16^{\star \star \star}$ & $8.91 \pm 6.05$ & $24.82 \pm 5.59^{\star \star \star}$ \\
\hline Soluble fiber, g & $5.71 \pm 2.96$ & $25.07 \pm 2.73^{\star \star \star}$ & $5.44 \pm 3.69$ & $23.45 \pm 3.06^{\star \star \star}$ \\
\hline
\end{tabular}

Data are means $\pm S D$. Paired t test (two-tailed), ${ }^{*} p<0.05,{ }^{* * *} p<0.001$ vs. week 0 in the same group; unpaired $t$-test (two-tailed), no significant difference between the A and $W$ group at the same time point. 
participants for 12 weeks. After the intervention, by design, both the two groups showed a significantly higher intake of dietary fibers compared with baseline. Participants in the $\mathrm{W}$ group also showed a significant decrease in fat intake from week 0 to week 12. The intakes of daily energy and macronutrient had no significant difference between the two groups at week 12 .

\section{Improvement of Clinical Parameters, Inflammatory Factors, and Brain-Gut Peptides in Patients With PCOS}

The clinical data of all participants (A group, $\mathrm{n}=11$; $\mathrm{W}$ group, $\mathrm{n}=14$ ) are summarized in Supplementary Table S1. Clinical parameters did not differ between the two groups at baseline.

The luteinizing hormone ( $\mathrm{LH}$ )/follicle-stimulating hormone (FSH) ratio of the A group showed a steady decrease trend during the intervention, significant decrease at week 12, and significant lower level compared to the $\mathrm{W}$ group at week 8 (Figure 2A). The testosterone level of the A group significantly decreased at weeks 4 and 8 , while the $\mathrm{W}$ group showed no significant change at this two time points and, in contrast, significantly increased at week 12 (Figure 2B). The hirsutism score of the A group was significantly lower after the intervention (Figure 2C). The result of transvaginal B-ultrasound showed that the ovarian volume and the number of immature follicles of the A group had a remarkable decrease after the intervention, while the $\mathrm{W}$ group had no change (Figures 2D, E). Three participants had unplanned pregnancy during the intervention and successfully delivered afterwards; especially, one of them in the A group had multiple failures of assisted reproductive technology in the past.

The abnormal level of blood glucose, hyperinsulinemia, and insulin resistance of the two groups were improved after the intervention (Figures $\mathbf{2 F}-\mathbf{H}$ and Supplementary Table S1). The A group had a better improvement of hyperinsulinemia and insulin resistance than the $\mathrm{W}$ group at weeks 4 and 8 . After the intervention, both of the two groups showed a significant decrease in BMI (Figure 2I) and blood lipid levels (Supplementary Table S1).

Markers of inflammation including LBP and $\alpha$-AGP decreased sharply at week 4 and maintained a stable level at

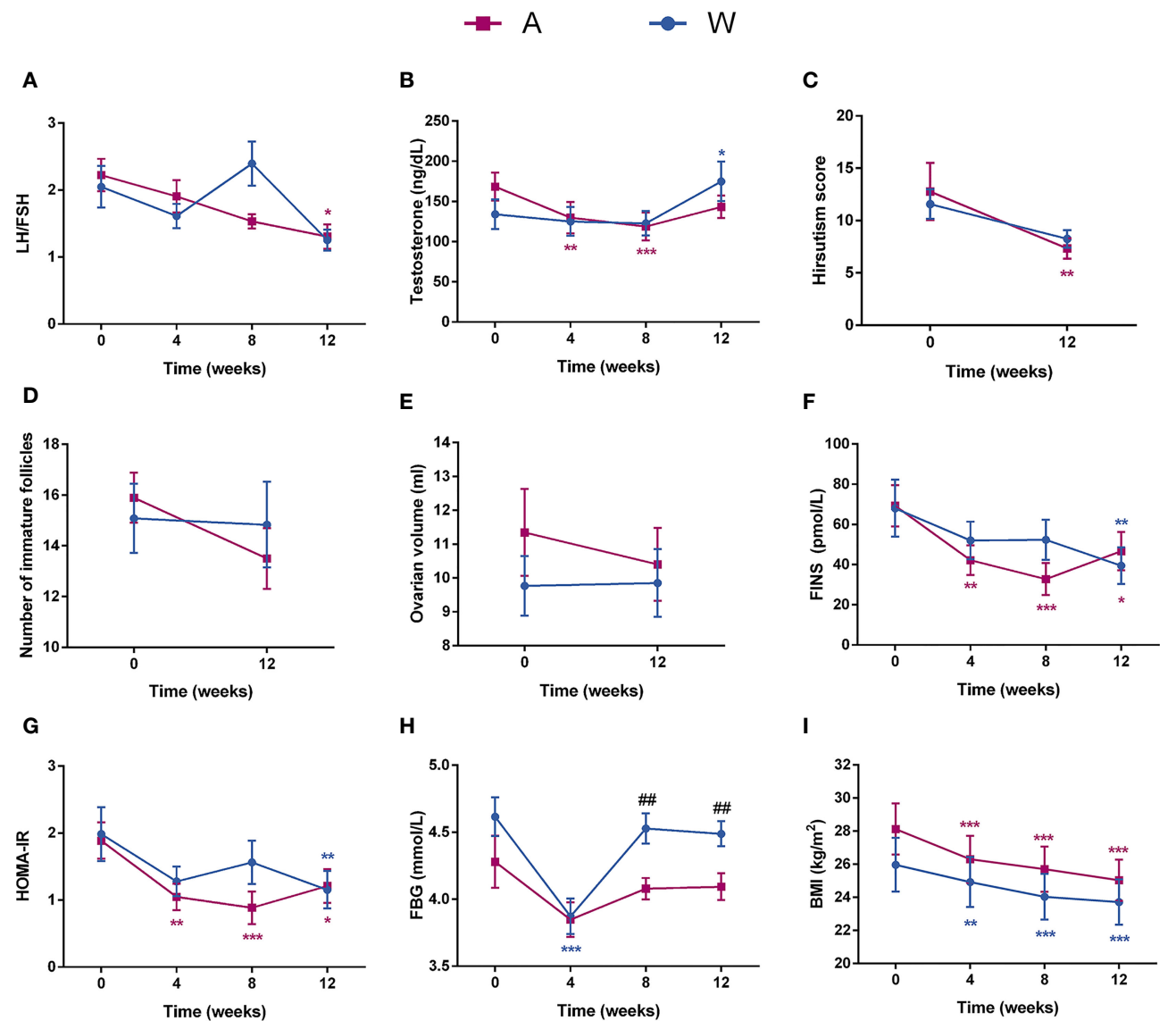

FIGURE 2 | The sex hormonal and metabolic disorders were improved in patients with PCOS after the intervention. Changes in (A) the LH/FSH ratio, (B) testosterone, (C) hirsutism score, (D) number of immature follicles, (E) ovarian volume, (F) FINS, (G) HOMA-IR, (H) FBG, and (I) BMl for participants during the intervention are shown. Date expressed as mean \pm SEM. ${ }^{*} p<0.05,{ }^{* *} p<0.01$, and ${ }^{\star * *} p<0.001$ for comparison with the week 0 value for the same group, red for $A$ group and blue for W group. ${ }^{\# \#} p<0.01$ for comparison of A group with W group value at the same time point. $\mathrm{LH}$, luteinizing hormone; FSH, follicular stimulating hormone; FINS, fasting plasma insulin; HOMA-IR, homeostasis model assessment for insulin resistance index; FBG, fasting blood glucose; BMl, body mass index. 
the two afterward visits in the two groups (Figures 3A, B). Meanwhile, the level of adiponectin, an anti-inflammatory cytokine, was significantly and steadily increased (Figure 3C). The level of spexin showed a significant trend of increase in the A group and was significantly higher than in the $\mathrm{W}$ group after intervention (Figure 3D). Orexin, a hypocretin peptide, was significantly decreased in the A group after intervention (Figure 3E). The serum leptin level of both groups decreased significantly at week 4 and maintain a stable level at the two afterward visits (Figure 3F).

\section{Altered Gut Microbiota by High-Fiber Diet or Combined With Acarbose}

Gene sequencing on the V3-V4 regions of the 16S rRNA generated a dataset consisting of 2,178,945 high-quality reads and 1,731 ASVs, with an average of $21,789 \pm 6,247$ reads per sample. According to the observed ASVs and the Shannon index, the richness of gut microbiota was significantly reduced in both groups and lower in the A group than in the $\mathrm{W}$ group after intervention (Figures 4A, B).

In the context of beta diversity based on Bray-Curtis (Figures 4C, D) and weighted-Unifrac (Figures 4E, F) distances, the overall microbial structure showed no difference between the two groups at baseline. Significant alterations were observed from baseline to week 4 in both groups, with no further changes afterwards. These results showed that the two sets of intervention both modulated the gut microbiota of patients with PCOS, and such modulation was stable. At weeks 4 until 12, especially at week 8, significant separation between the two groups reflected a distinct modulatory effect of the high-fiber diet combined with acarbose on the gut microbiota, compared to the simple high-fiber diet.

As bacteria work together as a coherent functional group in the gut ecosystem (31), we constructed a co-abundance network between the 132 ASVs, which were shared by at least $20 \%$ of all samples, and clustered the ASVs into 14 CAGs (Figure 5A and Supplementary Table S3). Of these, three CAGs, including CAG3, CAG7, and CAG11, were significantly increased in the A group, while two CAGs, including CAG3 and CAG7, were significantly increased in the $\mathrm{W}$ group. Six CAGs, including CAG4, CAG8, CAG9, CAG10, CAG12, and CAG14, were significantly decreased in the A group, while four CAGs, including CAG8, CAG9, CAG10, and CAG12, were significantly decreased in the $\mathrm{W}$ group. Six CAGs, including CAG4, CAG6, CAG7, CAG8, CAG11, and CAG14, responded to the differentiation between the two groups at weeks 4,8 , and 12 , five of which were also significantly altered in the A or W group. Max member of CAGs differed at week 4 between the two groups. CAG11 remain different at weeks 4, 8, and 12 (Figure 5B).

Among these CAGs, CAG3, and CAG11, which were enriched in the A group, were the dominant CAGs with ASVs from Bifidobacterium and Lactobacillus. CAG7 was $66.3 \%$ comprised predominantly of ASV1077 from Fusicatenibacter. CAG8, inhibited in both groups, was comprised predominantly of Bacteroides vulgatus and Alistipes. The other CAGs, inhibited in both groups, included CAG12 composed of ASVs from Bacteroides and Blautia, CAG10 composed of ASVs from Lachnospira and Roseburia, and CAG9 composed of ASVs
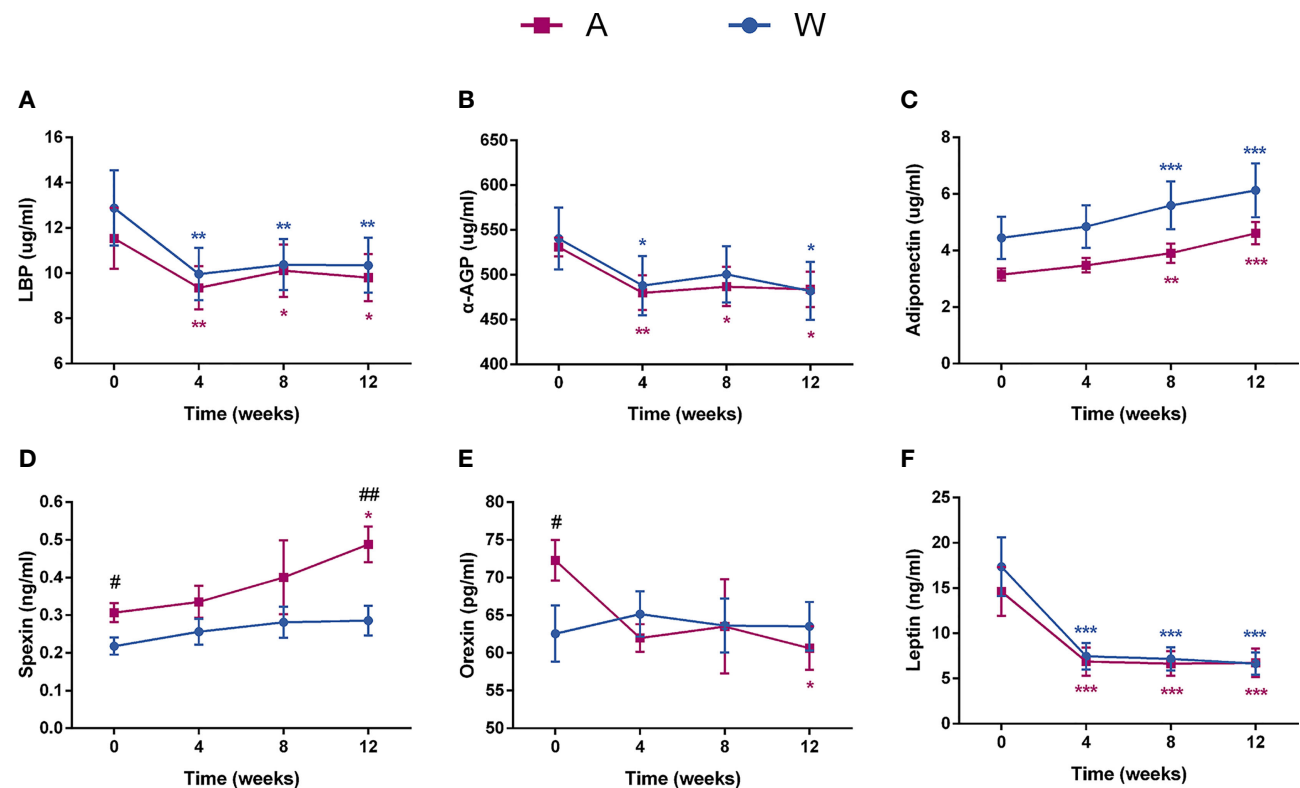

FIGURE 3 | The state of chronic low-grade inflammation and the secretion of brain-gut peptides were altered in patients with PCOS after the intervention. Serum (A) LBP, (B) $\alpha$-AGP, (C) adiponectin, (D) spexin, (E) orexin, and (F) leptin levels were tested by ELISA. Date expressed as mean \pm SEM. ${ }^{\star} p<0.05$, ${ }^{\star *} p<0.01$, and ${ }^{* * *} p<0.001$ for comparison with the week 0 value for the same group, red for A group and blue for W group. ${ }^{\#} p<0.05$ and ${ }^{\# \#} p<0.01$ for comparison of $A$ group with W group value at the same time point. LBP, lipopolysaccharide -binding protein; $\alpha$-AGP, $\alpha$-1-acid glycoprotein. 
A

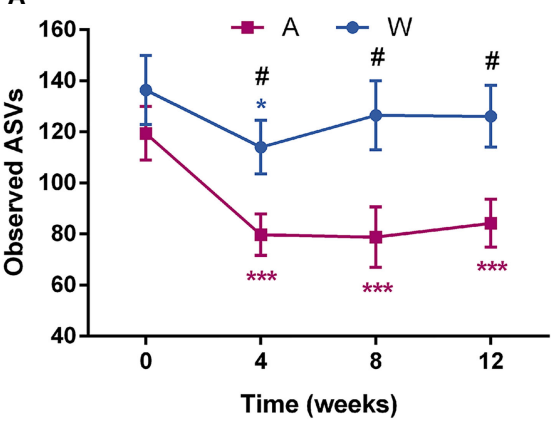

C

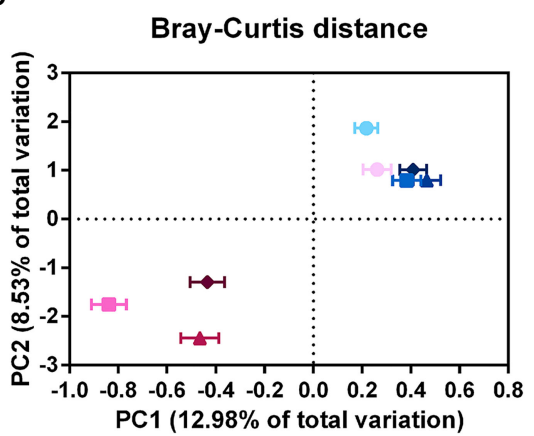

E

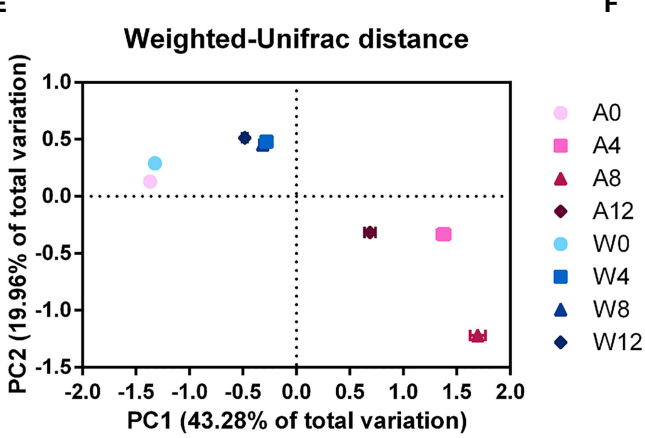

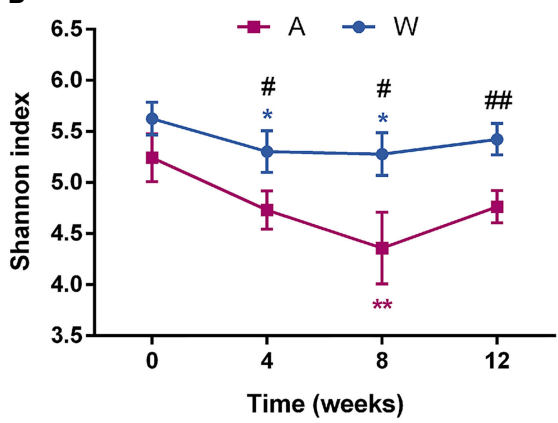
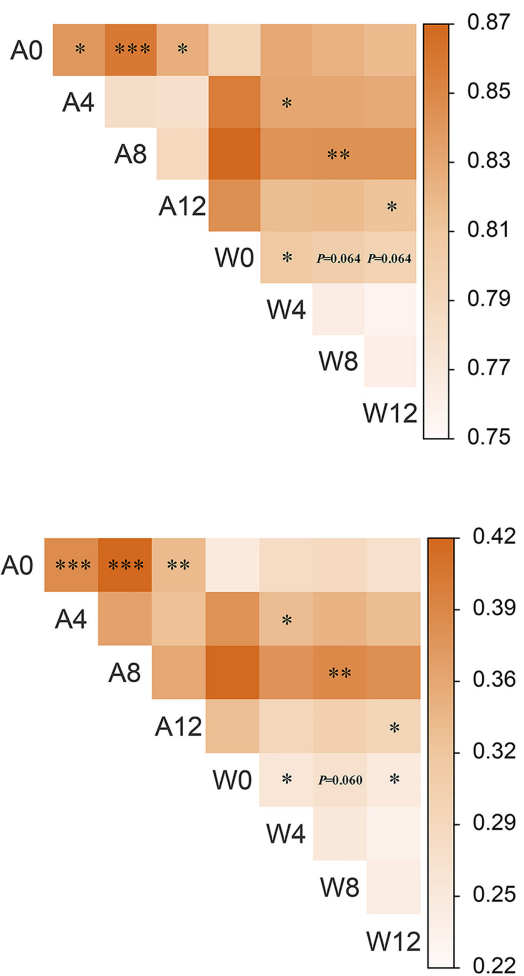

FIGURE 4 | The gut microbial structure was altered in patients with PCOS after the intervention. Alpha diversity measured by (A) observed ASVs and (B) Shannon index. Date expressed as mean \pm SEM. ${ }^{*} p<0.05,{ }^{* *} p<0.01$, and ${ }^{* *} p<0.001$ for comparison with the week 0 value for the same group, red for $A$ group and blue for $W$ group. ${ }^{\#} p<0.05$ and ${ }^{\# \#} p<0.01$ for comparison of $A$ group with $W$ group value at the same time point. Principal coordinates analysis (PCoA) performed on the basis of the (C) Bray-Curtis and (E) weighted-Unifrac distances showed the overall changes in gut microbial structure. PC1, principal coordinate 1; PC2, principal coordinate 2. PERMANOVA test was performed on the basis of the (D) Bray-Curtis and (F) weighted-Unifrac distances. The colors of the blocks indicate the distance, and the asterisks denote significant difference between two time points or different groups. ${ }^{*} p<0.05,{ }^{* *} p<0.01$, and ${ }^{\star \star *} p<0.001$. A0, A4, A8, and A12: weeks 0,4 , 8, and 12 in the A group; WO, W4, W8, and W12: weeks $0,4,8$, and 12 in the W group.

from Bilophila. CAG14, inhibited only in the A group, contained three ASVs from B. vulgatus.

\section{Associations Between Gut Microbiota and Clinical Parameters in Patients With PCOS}

To explore the relationships between the altered CAGs and host clinical phenotypes, we used MaAslin2 to get the correlations between the CAGs and 26 clinical parameters in different cohorts (Figure 6). When examining the whole cohort or A group separately, we saw that the CAGs enriched by the intervention were negatively correlated with the disease phenotypes, such as the LH/FSH ratio, testosterone, metabolic parameters including FINS and homeostasis model assessment-insulin resistance (HOMA-IR), $\alpha$-AGP, leptin, and orexin, and positively correlated with adiponectin and spexin, while the CAGs inhibited had an opposite trend (Figures 6A, B). Compared with the A group, the CAGs altered in the $\mathrm{W}$ group had the same trend but with weaker correlation with the disease phenotypes, mainly including the LH/FSH ratio, testosterone, the hirsutism score, FINS, and HOMA-IR (Figure 6C). 


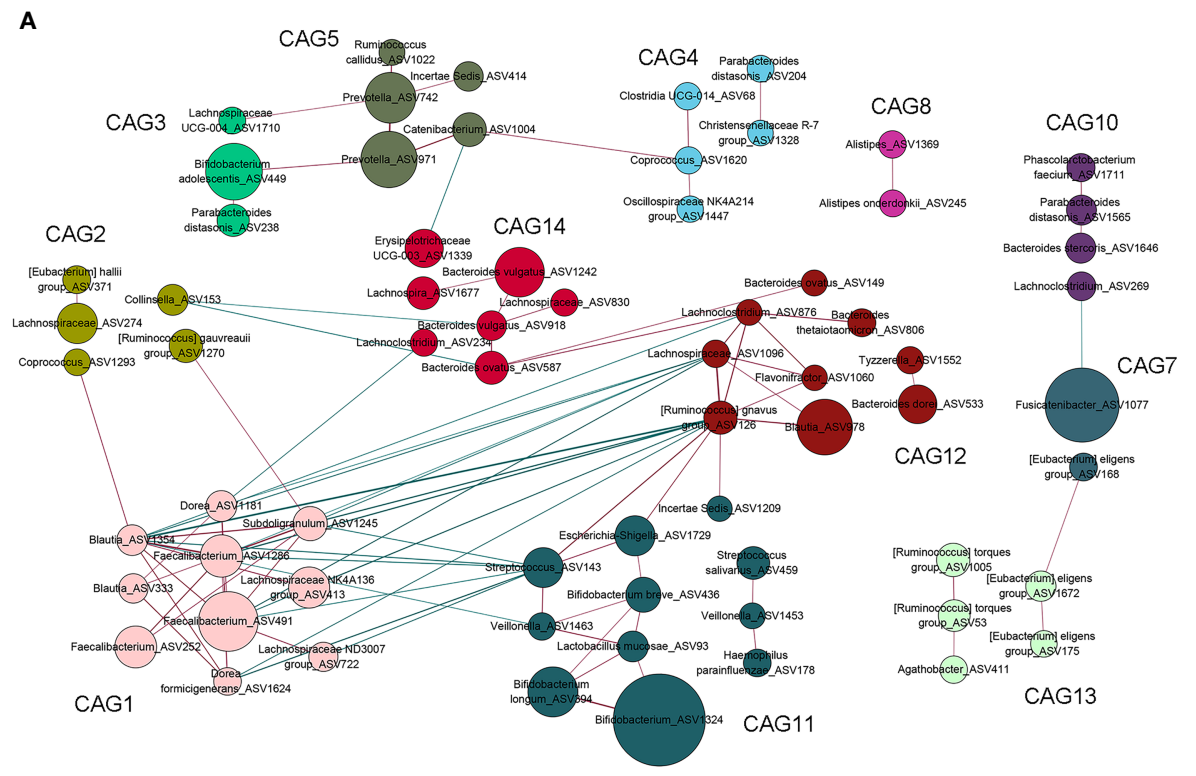

B

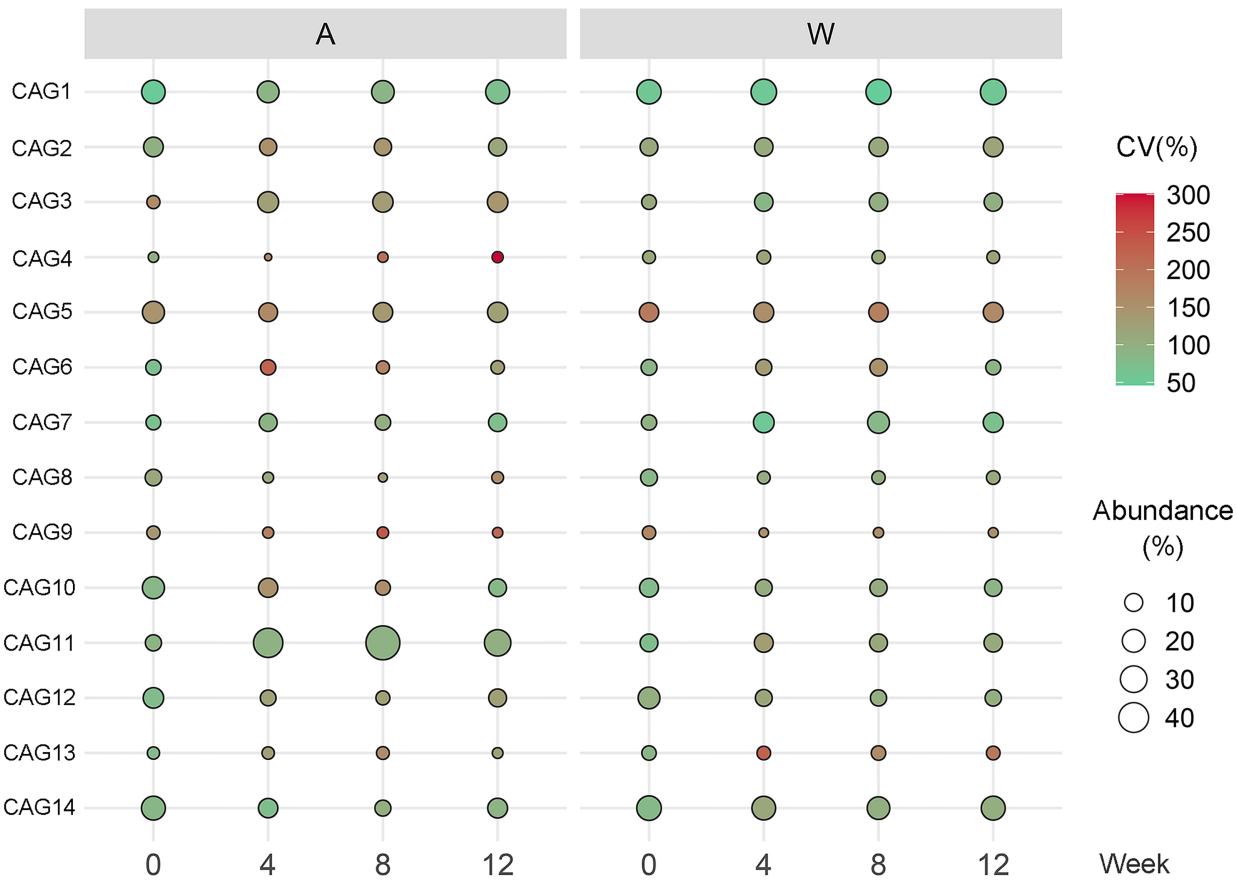

FIGURE 5 | Alterations in the abundance of CAGs in response to the intervention. (A) Co-abundance groups interaction network displays the interaction between different CAGs. Node size represents the average abundance of each ASV. Lines between the nodes indicate correlation (green = negative, red = positive), with width of the lines representing the correlation magnitude. Correlations with absolute values $<0.4$ are not shown here. (B) Bubble plot shows the variation in the average abundance of CAGs during the intervention. The size and color of the circles represent the average abundance and coefficient of variance (CV) of each CAG, respectively.

\section{DISCUSSION}

PCOS is a clinical syndrome with metabolic, endocrine, and reproductive dysfunctions. Dietary interventions as a first-line treatment for patients with PCOS have been evaluated, but the optimal diet pattern is uncertain. Effective treatments for PCOS are still lacking. Our clinical trial showed that high-fiber diet or combined with acarbose alleviate PCOS by regulating gut microbiota. In this trial, we conducted a 12 -week clinical intervention in patients with PCOS, achieved the improvement 

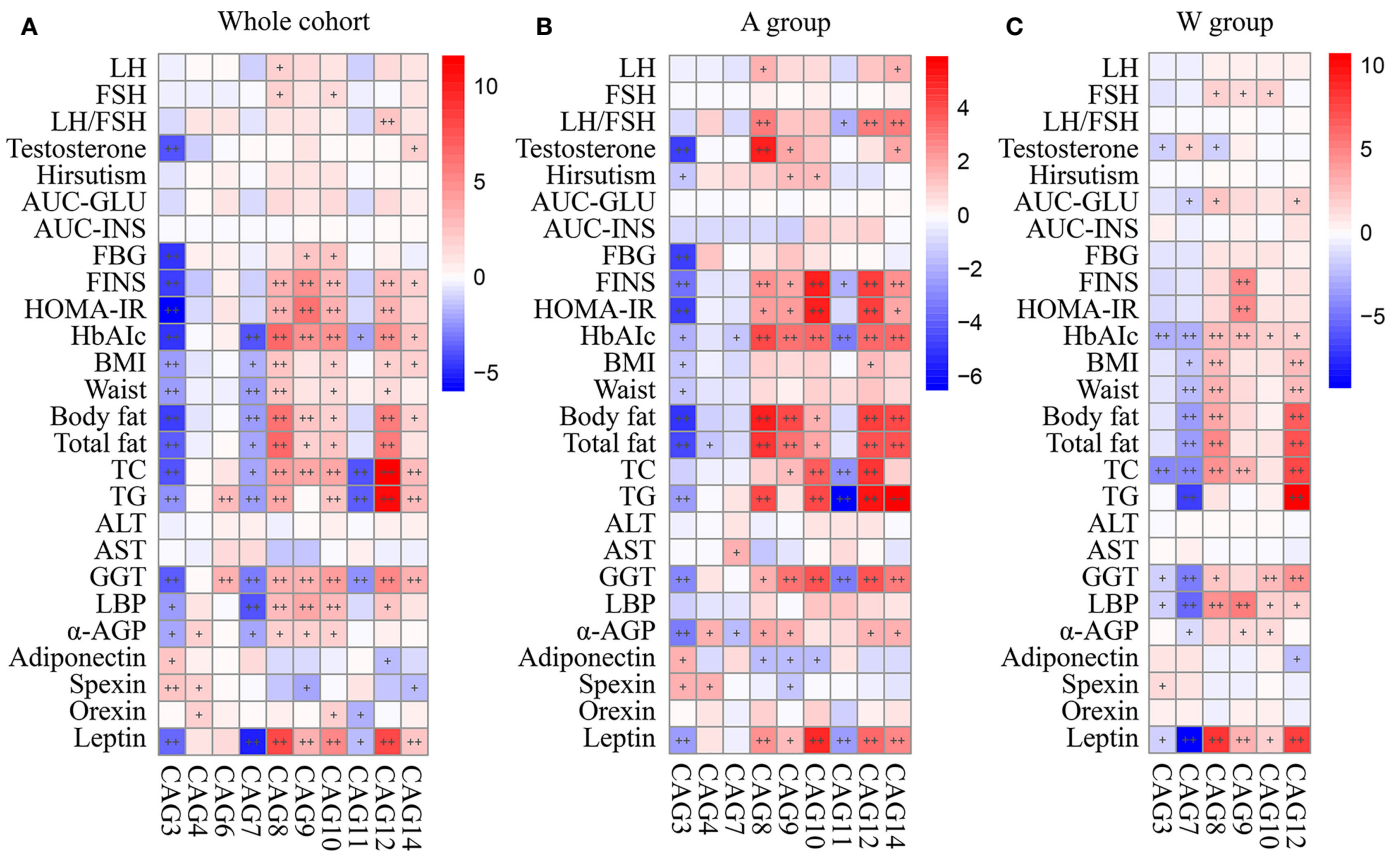

FIGURE 6 | Correlations between primary clinical parameters and altered CAGs in (A) the whole cohort, (B) A group, and (C) W group. In the heat map, spots color represents R-value of MaAslin2 correlation between each CAG and clinical parameter. ${ }^{+} \mathrm{FDR}<0.25$ and ${ }^{++} \mathrm{FDR}<0.1$. LH, luteinizing hormone; FSH, follicular stimulating hormone; AUC-Glucose, the glucose area under the curve of OGTT; AUC-Insulin, the insulin area under the curve of OGTT; FBG, fasting blood glucose; FINS, fasting plasma insulin; HOMA-IR, homeostasis model assessment for insulin resistance index; HbA1c, hemoglobin A1c; BMl, body mass index; TC, total cholesterol; TG, triglyceride; ALT, alanine aminotransferase; AST, aspartate transaminase; GGT, $\gamma$-glutamyltransferase; LBP, lipopolysaccharide-binding protein; $\alpha$-AGP, $\alpha$-1-acid glycoprotein.

of reproductive endocrine and glycolipid metabolic disorders, and promoted fertility via intervention of no matter simple highfiber diet or combined with acarbose. As far as we know, this trial is the first gut microbiota-targeted nutritional intervention combined with oral hypoglycemic agent for the patients with PCOS. The dysbiosis of gut microbiota was remodeled by a highfiber diet, probiotics were enriched, and noxious bacteria were inhibited, thus leading to the changes in $\mathrm{pH}$ level and short-chain fatty acids (SCFAs) production (Supplementary Figure S1). It is worth noting that a high-fiber diet combined with acarbose showed the better improvement of reproductive endocrine disorders, hyperinsulinemia and insulin resistance, and more optimal composition of the gut microbiota. Furthermore, the changes in gut microbiota and SCFAs were associated with sex hormonal and metabolic parameters, inflammatory factors, and brain-gut peptides, especially in the A group.

Obesity and insulin resistance are strongly implicated in the etiology of PCOS, and lifestyle modification, especially dietary modification and exercise, is the primary method to improve insulin sensitivity (3). Weight loss leads to the improvements in the health of overweight/obese women with PCOS $(32,33)$. Dietary fiber supplementation has a definite effect on weight loss (34). The intake of dietary fibers is also associated with insulin resistance, hyperandrogenism, and body composition in patients with PCOS $(7,35)$. In our study, both of the two groups were given a high-fiber diet and showed a significant reduction in
BMI, which may alleviate the pathophysiological defects of PCOS. Moreover, the structure of gut microbiota changed significantly after high-fiber diet intervention compared with baseline. The dietary fibers we provided was a single staple food, and this single diet led to the reduction in richness of gut microbiota. Our data suggest that optimal structure of the gut microbiota rather than simple greater overall diversity implies better health, which is consistent with our previous study (18). Besides, dietary fibers could promote the production of key metabolites such as SCFAs, impacting host-microbe interactions $(18,36)$, and this mechanism might explain our results about the SCFAs. Furthermore, SCFAs could combat obesity and insulin resistance, a core pathogenic factor of PCOS, by brain-gut peptides (37). Therefore, it suggests that dietary fibers may alleviate the clinical phenotypes of PCOS, and the gut microbiota may play an important role in the process.

Acarbose could push more carbohydrates into the large intestine for fermentation. This mechanism confirmed the modulation of acarbose on gut microbiota (38). However, a previous study found that acarbose combined with another kind of high-fiber diet promoted more probiotics than acarbose alone (18), in relative agreement with our finding that acarbose could strengthen the modulation of dietary fibers on gut microbiota. We found that the high-fiber diet combined with acarbose could better improve the hyperandrogenism and insulin resistance of host, which suggested that acarbose alleviates PCOS by the two 
core pathogenic factors. However, it seemed to be due to the participants neglect of their diet from the third month; these improvements were rebounded slightly at the end of our intervention, and this trend was consistent with changes in gut microbiota. However, the specific mechanism remains to be further studied.

Multiple inflammatory factors and brain-gut peptides may be involved in the development of PCOS. LBP, known as LPSbinding protein, could affect insulin sensitivity and lead to IR (39). Zhu et al. found that LBP level in patients with PCOS was significantly increased, irrespective of body mass (40). $\alpha$-AGP and adiponectin are adipokines, belonging to the family of cytokines and inflammatory proteins that are produced by adipose tissue $(41,42)$. $\alpha$-AGP may modulate energy homeostasis and food intake by interacting with leptin receptor (43). In PCOS, $\alpha$-AGP level was higher and correlated with biomarkers of adiposity and total testosterone (44). Adiponectin mediates insulin-sensitizing effect through binding to its receptors AdipoR1 and AdipoR2 (42). Spexin is a novel hypothalamic neuropeptide that exerts inhibitory effect on feeding and maintains reproductive function via gonadotropinreleasing hormone neurons (45-47). The decreased level of spexin is associated with sex hormonal and metabolic disorders of PCOS (48). Orexin is another hypothalamic neuropeptide and has been demonstrated to evoke hyperphagia and obesity (49). Leptin is involved in gut-brain axis, thus regulating appetite and energy metabolism $(50,51)$. Leptin, adiponectin, and the leptin/ adiponectin ratio are correlated with insulin resistance (52). Our study showed that patients of PCOS had a significant decrease in LBP, $\alpha$-AGP, and leptin, and significant increase in adiponectin after the intervention. Spexin significantly increased and orexin significantly decreased in the intervention added of acarbose. Our results indicate that the intervention may affect the levels of inflammatory factors and brain-gut peptides and thus alleviate the clinical phenotypes of PCOS.

A previous study slightly focused on the beneficial effects of prebiotics in patients with PCOS. Dietary fibers and acarbose may affect the gut microbiota structure in the form of CAGs, rather than single bacteria. Here, we observed that CAGs, comprised predominantly of Bifidobacterium and Fusicatenibacter, were enriched after the intervention in both of the two groups. Ren et al. (53) reported that the decrease in the abundance of Fusicatenibacter in non-alcoholic fatty liver disease was linked with the metabolism disorders such as insulin resistance. According to the report by Takada et al. (54), Fusicatenibacter could ferment carbohydrates to produce SCFAs. It should be noted that high-fiber diet combined with acarbose enriched large numbers of Bifidobacterium and a strain of Lactobacillus than simple high-fiber diet. Bifidobacterium could ferment carbohydrates including mannose, fructose, sucrose, and lactose (55). More studies showed that the fermentation of prebiotics including inulin by Bifidobacterium and Lactobacillus promoted host health $(56,57)$. The results of our study suggest that acarbose promote more carbohydrates into the large intestine for Bifidobacterium and Lactobacillus consumption and thus lead to the enrichment of ASVs in the A group. According to previous studies, Bifidobacterium and Lactobacillus genera are rich in acetic-acid-producing bacteria, which is in agreement with the acetic change in our result (Supplemetary Figure S1). In our study, CAG3 and CAG11, mainly containing Bifidobacterium, had a negative correlation with the levels of LH/FSH ratio, testosterone, glycolipid metabolism, inflammatory factors, orexin, and leptin, while they were positive correlated with the levels of adiponectin and spexin. Zhang et al. (14) proved a direct evidence that Bifidobacterium lactis V9 regulates the levels of sex hormones in PCOS via the gut-brain axis. CAG7, mainly containing Fusicatenibacter, had a negative correlation with the glycolipid metabolism parameters, inflammatory factors, and leptin. These results suggest that the enrichment of these bacteria may alter the chronic metabolic inflammation and brain-gut peptides of host and thus alleviate PCOS by affecting the SCFAs production.

On the other hand, our intervention also decreased the relative abundance of several CAGs, comprised predominantly of noxious bacteria, such as Bacteroides, mainly of B. vulgatus, and Alistipes, Bilophila, Blautia, Lachnospira, and Roseburia. It should be noted that a high-fiber diet combined with acarbose inhibited more ASVs from B. vulgatus than a simple high-fiber diet. CAG8 and CAG14, mainly containing B. vulgatus, were stronger inhibited in the A group and had a positive correlation with the levels of LH/FSH ratio, testosterone, hyperinsulinemia, insulin resistance, BMI, inflammatory factors, and leptin, while they were negatively correlated with the levels of adiponectin and spexin. Hence, it can be seen that acarbose strengthened the modulation of gut microbiota by a high-fiber diet and might better alleviate PCOS through the two core pathogenic factors, namely, hyperandrogenism and insulin resistance. Bacteroides in the human intestine are Gram-negative bacteria, a kind of famous LPS-producing bacteria (58). The positive correlation of Bacteroides and metabolism disorders in PCOS from a crosssectional study confirmed our result (10). Sun et al. (59) and Jiang et al. (60) elaborated our results in the mechanism; they pointed out that Bacteroides fragilis and B. vulgatus affected the host metabolic disorders and inflammation of T2DM and PCOS via the gut microbiota-bile acid axis. Besides, CAG8 also contained ASVs from Alistipes. Alistipes, another kind of common LPS-producing bacteria, is associated with obesity and inflammation and can aggravate metabolism disorders induced by high-fat diet (61). Other CAGs, such as CAG9, CAG10, and CAG12, containing ASVs from Bacteroides, Bilophila, Blautia, and Roseburia, were inhibited in both of the two groups and had a positive correlation with a series of glycolipid metabolism parameters, inflammatory factors, orexin, and leptin, while they were negatively correlated with adiponectin and spexin. Bilophilia, another common LPSproducing bacteria, could promote higher inflammation and bile acid dysmetabolism, thus aggravating the metabolic dysfunctions induced by high-fat diet (62). Lin et al. reported an increase in the abundance of Blautia genera, and Roseburia genera are correlated with the alterations of bile acids in obesity (63). These results suggest that the inhibition of these bacteria may improve the chronic metabolic inflammation by 
affecting the LPS production and bile acid metabolism, thus alleviating PCOS.

Our study was a pilot exploration of dietary or drug interventions targeting gut microbiota in patients with PCOS. The downside of our study is the small sample size. Due to the strict quality control of the experimental process, the dietary intervention programs of all subjects were consistent, and the changes in physiological and biochemical parameters and gut microbiota showed the same trend. Further multicenter, placebocontrolled, double-blind studies, metabonomic studies, and fecal microbiota transplantation in germ-free mice are needed to be performed to explore more mechanisms of how the gut microbiota modulation alleviate PCOS.

In summary, our work suggested that high-fiber diet could alleviate the chronic metabolic inflammation, reproductive function, and brain-gut peptides secretion of patients with PCOS, and a high-fiber diet combined with acarbose could better improve the PCOS clinical phenotypes. Meanwhile, the remodeling of gut microbiota by our intervention may play an important role in these improvements. Gut microbiota targeted intervention may present a novel approach for the treatment of PCOS in clinical practice.

\section{DATA AVAILABILITY STATEMENT}

The original contributions presented in the study are included in the article/Supplementary Material, further inquiries can be directed to the corresponding authors.

\section{ETHICS STATEMENT}

The studies involving human participants were reviewed and approved by the Human Research Ethics Committee of Shanghai General Hospital. Written informed consent to participate in this study was provided by the participants' legal guardian/next of kin.

\section{REFERENCES}

1. Rotterdam ESHRE/ASRM-Sponsored PCOS Consensus Workshop Group. Revised 2003 Consensus on Diagnostic Criteria and Long-Term Health Risks Related to Polycystic Ovary Syndrome (PCOS). Hum Reprod (2004) 19(1):417. doi: 10.1093/humrep/deh098

2. Meier RK. Polycystic Ovary Syndrome. Nurs Clin North Am (2018) 53 (3):407-20. doi: 10.1016/j.cnur.2018.04.008

3. Wang J, Wu D, Guo H, Li M. Hyperandrogenemia and Insulin Resistance: The Chief Culprit of Polycystic Ovary Syndrome. Life Sci (2019) 236:116940. doi: 10.1016/j.lfs.2019.116940

4. Douglas CC, Norris LE, Oster RA, Darnell BE, Azziz R, Gower BA. Difference in Dietary Intake Between Women With Polycystic Ovary Syndrome and Healthy Controls. Fertil Steril (2006) 86(2):411-7. doi: 10.1016/j.fertnstert.2005.12.054

5. Altieri P, Cavazza C, Pasqui F, Morselli AM, Gambineri A, Pasquali R. Dietary Habits and Their Relationship With Hormones and Metabolism in Overweight and Obese Women With Polycystic Ovary Syndrome. Clin Endocrinol (Oxf) (2013) 78(1):52-9. doi: 10.1111/j.1365-2265.2012.04355.x

\section{AUTHOR CONTRIBUTIONS}

XWa performed clinical research, DNA extraction and sequencing, and the bioinformatics and statistical analysis. TX performed the bioinformatics and statistical analysis. RL performed DNA extraction and SCFA measurements. GW performed the bioinformatics analysis. LG and YLi collected clinical data. YZ, XWe, and YLu performed statistical analysis. FZ and JS performed clinical research. HF performed nutritional intervention. LZ, YP, and CZ designed the study and performed quality control. XD designed the study, performed clinical research, and wrote the manuscript. All authors contributed to the article and approved the submitted version.

\section{FUNDING}

The study was supported by the National Natural Science Foundation of China (81870594); Shanghai Jiao Tong University Research Funding on Medical, Engineering Interdisciplinary Project (YG2019GD05); Clinical Research Plan of SHDC (SHDC2020CR1016B); Multi-center Clinical Research Project of Shanghai Jiao Tong University School of Medicine (DLY201824); The Third Round Cooperation Project of Songjiang District Municipal Health Commission (0702N18003).

\section{ACKNOWLEDGMENTS}

We are grateful to all the participants, medical staffs, and researchers in the study.

\section{SUPPLEMENTARY MATERIAL}

The Supplementary Material for this article can be found online at: https://www.frontiersin.org/articles/10.3389/fendo.2021. 806331/full\#supplementary-material

6. Barrea L, Arnone A, Annunziata G, Muscogiuri G, Laudisio D, Salzano C, et al. Adherence to the Mediterranean Diet, Dietary Patterns and Body Composition in Women With Polycystic Ovary Syndrome (PCOS). Nutrients (2019) 11(10):2278. doi: 10.3390/nu11102278

7. Cutler DA, Pride SM, Cheung AP. Low Intakes of Dietary Fiber and Magnesium are Associated With Insulin Resistance and Hyperandrogenism in Polycystic Ovary Syndrome: A Cohort Study. Food Sci Nutr (2019) 7 (4):1426-37. doi: 10.1002/fsn3.977

8. Proctor C, Thiennimitr P, Chattipakorn N, Chattipakorn SC. Diet, Gut Microbiota and Cognition. Metab Brain Dis (2017) 32(1):1-17. doi: 10.1007/s11011-016-9917-8

9. Ojeda P, Bobe A, Dolan K, Leone V, Martinez K. Nutritional Modulation of Gut Microbiota - the Impact on Metabolic Disease Pathophysiology. J Nutr Biochem (2016) 28:191-200. doi: 10.1016/j.jnutbio.2015.08.013

10. Liu R, Zhang C, Shi Y, Zhang F, Li L, Wang X, et al. Dysbiosis of Gut Microbiota Associated With Clinical Parameters in Polycystic Ovary Syndrome. Front Microbiol (2017) 8:324. doi: 10.3389/fmicb.2017.00324

11. Jobira B, Frank DN, Pyle L, Silveira LJ, Kelsey MM, Garcia-Reyes Y, et al. Obese Adolescents With PCOS Have Altered Biodiversity and Relative 
Abundance in Gastrointestinal Microbiota. J Clin Endocrinol Metab (2020) 105(6):e2134-44. doi: 10.1210/clinem/dgz263

12. Insenser M, Murri M, Del Campo R, Martinez-Garcia MA, Fernandez-Duran E, Escobar-Morreale HF. Gut Microbiota and the Polycystic Ovary Syndrome: Influence of Sex, Sex Hormones, and Obesity. J Clin Endocrinol Metab (2018) 103(7):2552-62. doi: 10.1210/jc.2017-02799

13. Zeng B, Lai Z, Sun L, Zhang Z, Yang J, Li Z, et al. Structural and Functional Profiles of the Gut Microbial Community in Polycystic Ovary Syndrome With Insulin Resistance (IR-PCOS): A Pilot Study. Res Microbiol (2019) 170(1):4352. doi: 10.1016/j.resmic.2018.09.002

14. Zhang J, Sun Z, Jiang S, Bai X, Ma C, Peng Q, et al. Probiotic Bifidobacterium Lactis V9 Regulates the Secretion of Sex Hormones in Polycystic Ovary Syndrome Patients Through the Gut-Brain Axis. mSystems (2019) 4(2): e00017-19. doi: 10.1128/mSystems.00017-19

15. Karamali M, Eghbalpour S, Rajabi S, Jamilian M, Bahmani F, TajabadiEbrahimi M, et al. Effects of Probiotic Supplementation on Hormonal Profiles, Biomarkers of Inflammation and Oxidative Stress in Women With Polycystic Ovary Syndrome: A Randomized, Double-Blind, Placebo-Controlled Trial. Arch Iran Med (2018) 21(1):1-7 https://pubmed.ncbi.nlm.nih.gov/29664663/.

16. Ahmadi S, Jamilian M, Karamali M, Tajabadi-Ebrahimi M, Jafari P, Taghizadeh M, et al. Probiotic Supplementation and the Effects on Weight Loss, Glycaemia and Lipid Profiles in Women With Polycystic Ovary Syndrome: A Randomized, Double-Blind, Placebo-Controlled Trial. Hum Fertil (Camb) (2017) 20(4):254-61. doi: 10.1080/14647273.2017.1283446

17. Soare A, Khazrai YM, Del Toro R, Roncella E, Fontana L, Fallucca S, et al. The Effect of the Macrobiotic Ma-Pi 2 Diet vs. The Recommended Diet in the Management of Type 2 Diabetes: The Randomized Controlled MADIAB Trial. Nutr Metab (Lond) (2014) 11:39. doi: 10.1186/1743-7075-11-39

18. Zhao L, Zhang F, Ding X, Wu G, Lam YY, Wang X, et al. Gut Bacteria Selectively Promoted by Dietary Fibers Alleviate Type 2 Diabetes. Science (2018) 359(6380):1151-6. doi: 10.1126/science.aao5774

19. Turner-Mcgrievy G, Davidson CR, Billings DL. Dietary Intake, Eating Behaviors, and Quality of Life in Women With Polycystic Ovary Syndrome Who are Trying to Conceive. Hum Fertil (Camb) (2015) 18(1):16-21. doi: 10.3109/14647273.2014.922704

20. Mccarty MF, Dinicolantonio JJ. Acarbose, Lente Carbohydrate, and Prebiotics Promote Metabolic Health and Longevity by Stimulating Intestinal Production of GLP-1. Open Heart (2015) 2(1):e000205. doi: 10.1136/ openhrt-2014-000205

21. Su B, Liu H, Li J, Sunli Y, Liu B, Liu D, et al. Acarbose Treatment Affects the Serum Levels of Inflammatory Cytokines and the Gut Content of Bifidobacteria in Chinese Patients With Type 2 Diabetes Mellitus. J Diabetes (2015) 7(5):729-39. doi: 10.1111/1753-0407.12232

22. Zhang YY, Hou LQ, Zhao TY. Effects of Acarbose on Polycystic Ovary Syndrome: A Meta-Analysis. Exp Clin Endocrinol Diabetes (2014) 122(6):3738. doi: $10.1055 / \mathrm{s}-0034-1375676$

23. Zhao X, Jiang Y, Xi H, Chen L, Feng X. Exploration of the Relationship Between Gut Microbiota and Polycystic Ovary Syndrome (PCOS): A Review. Geburtshilfe Frauenheilkd (2020) 80(2):161-71. doi: 10.1055/a-1081-2036

24. Zhang C, Yin A, Li H, Wang R, Wu G, Shen J, et al. Dietary Modulation of Gut Microbiota Contributes to Alleviation of Both Genetic and Simple Obesity in Children. EBioMedicine (2015) 2(8):968-84. doi: 10.1016/j.ebiom.2015.07.007

25. Zhang Q, Wu Y, Wang J, Wu G, Long W, Xue Z, et al. Accelerated Dysbiosis of Gut Microbiota During Aggravation of DSS-Induced Colitis by a ButyrateProducing Bacterium. Sci Rep (2016) 6:27572. doi: 10.1038/srep27572

26. Bolyen E, Rideout JR, Dillon MR, Bokulich NA, Abnet CC, Al-Ghalith GA, et al. Reproducible, Interactive, Scalable and Extensible Microbiome Data Science Using QIIME 2. Nat Biotechnol (2019) 37(8):852-7. doi: 10.1038/ s41587-019-0209-9

27. Callahan BJ, Mcmurdie PJ, Rosen MJ, Han AW, Johnson AJ, Holmes SP. DADA2: High-Resolution Sample Inference From Illumina Amplicon Data. Nat Methods (2016) 13(7):581-3. doi: 10.1038/nmeth.3869

28. Bokulich NA, Kaehler BD, Rideout JR, Dillon M, Bolyen E, Knight R, et al. Optimizing Taxonomic Classification of Marker-Gene Amplicon Sequences With QIIME 2's Q2-Feature-Classifier Plugin. Microbiome (2018) 6(1):90. doi: 10.1186/s40168-018-0470-z

29. Quast C, Pruesse E, Yilmaz P, Gerken J, Schweer T, Yarza P, et al. The SILVA Ribosomal RNA Gene Database Project: Improved Data Processing and Web-
Based Tools. Nucleic Acids Res (2013) 41(Database issue):D590-596. doi: $10.1093 / \mathrm{nar} / \mathrm{gks} 1219$

30. Friedman J, Alm EJ. Inferring Correlation Networks From Genomic Survey Data. PloS Comput Biol (2012) 8(9):e1002687. doi: 10.1371/journal.pcbi.1002687

31. Wu G, Zhao N, Zhang C, Lam YY, Zhao L. Guild-Based Analysis for Understanding Gut Microbiome in Human Health and Diseases. Genome Med (2021) 13(1):22. doi: 10.1186/s13073-021-00840-y

32. Anuja D, Sarwer D, Allison KC, Lauren M, Kris-Etherton PM, Kunselman AR, et al. Weight Loss and Lowering Androgens Predict Improvements in Health-Related Quality of Life in Women With PCOS. J Clin Endocrinol Metab (2016) 8):2966-74. doi: 10.1210/jc.2016-1896

33. Glueck CJ, Goldenberg N. Characteristics of Obesity in Polycystic Ovary Syndrome: Etiology, Treatment, and Genetics. Metabolism (2019) 92:108-20. doi: 10.1016/j.metabol.2018.11.002

34. Thompson SV, Hannon BA, An R, Holscher HD. Effects of Isolated Soluble Fiber Supplementation on Body Weight, Glycemia, and Insulinemia in Adults With Overweight and Obesity: A Systematic Review and Meta-Analysis of Randomized Controlled Trials. Am J Clin Nutr (2017) 106(6):1514-28. doi: 10.3945/ajcn.117.163246

35. Cunha NBD, Ribeiro CT, Silva CM, Rosa ESA, De-Souza DA. Dietary Intake, Body Composition and Metabolic Parameters in Women With Polycystic Ovary Syndrome. Clin Nutr (2019) 38(5):2342-8. doi: 10.1016/j.clnu.2018.10.012

36. Makki K, Deehan EC, Walter J, Backhed F. The Impact of Dietary Fiber on Gut Microbiota in Host Health and Disease. Cell Host Microbe (2018) 23 (6):705-15. doi: 10.1016/j.chom.2018.05.012

37. Hernandez M, Canfora EE, Jocken J, Blaak E. The Short-Chain Fatty Acid Acetate in Body Weight Control and Insulin Sensitivity. Nutrients (2019) 11 (8):1943. doi: 10.3390/nul1081943

38. Zhang X, Fang Z, Zhang C, Xia H, Jie Z, Han X, et al. Effects of Acarbose on the Gut Microbiota of Prediabetic Patients: A Randomized, Double-Blind, Controlled Crossover Trial. Diabetes Ther (2017) 8(2):293-307. doi: 10.1007/ s13300-017-0226-y

39. Kim EK, Heo SJ, Han S, Kwon SK, Kim SY, Kim HJ, et al. Blood Concentrations of Lipopolysaccharide-Binding Protein, High-Sensitivity CReactive Protein, Tumor Necrosis Factor- $\alpha$, and Interleukin-6 in Relation to Insulin Resistance in Young Adolescents. Clin Chim Acta Int J Clin Chem (2018) 486:115-21. doi: 10.1016/j.cca.2018.07.042

40. Zhu Q, Huang Z, Zhang A, Gao R, Yang S, Zhao C, et al. Serum LBP Is Associated With Insulin Resistance in Women With PCOS. PloS One (2016) 11(1):e0145337. doi: 10.1371/journal.pone.0145337

41. Lee YS, Choi JW, Hwang I, Lee JW, Lee JH, Kim AY, et al. Adipocytokine Orosomucoid Integrates Inflammatory and Metabolic Signals to Preserve Energy Homeostasis by Resolving Immoderate Inflammation. J Biol Chem (2010) 285(29):22174-85. doi: 10.1074/jbc.M109.085464

42. Fang H, Judd RL. Adiponectin Regulation and Function. Compr Physiol (2018) 8(3):1031-63. doi: 10.1002/cphy.c170046

43. Sun Y, Yang Y, Qin Z, Cai J, Guo X, Tang Y, et al. The Acute-Phase Protein Orosomucoid Regulates Food Intake and Energy Homeostasis via Leptin Receptor Signaling Pathway. Diabetes (2016) 65(6):1630-41. doi: 10.2337/ $\mathrm{db} 15-1193$

44. De Medeiros SF, De Medeiros M, Barbosa BB, Yamamoto A, Yamamoto MMW. The Connection of Alpha-1 Acid Glycoprotein Inflammatory Marker With Anthropometric, Hormonal, and Metabolic Characteristic of Women With Polycystic Ovary Syndrome. J Obstet Gynaecol Res (2021) 47(10):357182. doi: $10.1111 /$ jog.14940

45. Liu Y, Li S, Qi X, Zhou W, Liu X, Lin H, et al. A Novel Neuropeptide in Suppressing Luteinizing Hormone Release in Goldfish, Carassius Auratus. Mol Cell Endocrinol (2013) 374(1-2):65-72. doi: 10.1016/j.mce.2013.04.008

46. Wang L, Tran A, Lee J, Belsham DD. Palmitate Differentially Regulates Spexin, and its Receptors Galr2 and Galr3, in GnRH Neurons Through Mechanisms Involving PKC, MAPKs, and TLR4. Mol Cell Endocrinol (2020) 518:110991. doi: 10.1016/j.mce.2020.110991

47. Coutinho EA, Prescott M, Hessler S, Marshall CJ, Herbison AE, Campbell RE. Activation of a Classic Hunger Circuit Slows Luteinizing Hormone Pulsatility. Neuroendocrinology (2020) 110(7-8):671-87. doi: 10.1159/000504225

48. Guler A, Demir I. Decreased Levels of Spexin Are Associated With Hormonal and Metabolic Disturbance in Subjects With Polycystic Ovary Syndrome. J Obstet Gynaecol (2021) 41(3):408-13. doi: 10.1080/01443615.2020.1737660 
49. Imperatore R, Palomba L, Cristino L. Role of Orexin-A in Hypertension and Obesity. Curr Hypertens Rep (2017) 19(4):34. doi: 10.1007/s11906-017-0729-y

50. Grosshans M, Vollmert C, Vollstadt-Klein S, Tost H, Leber S, Bach P, et al. Association of Leptin With Food Cue-Induced Activation in Human Reward Pathways. Arch Gen Psychiatry (2012) 69(5):529-37. doi: 10.1001/ archgenpsychiatry.2011.1586

51. Zanchi D, Depoorter A, Egloff L, Haller S, Mahlmann L, Lang UE, et al. The Impact of Gut Hormones on the Neural Circuit of Appetite and Satiety: A Systematic Review. Neurosci Biobehav Rev (2017) 80:457-75. doi: 10.1016/ j.neubiorev.2017.06.013

52. Frithioff-Bøjsøe C, Lund M, Lausten-Thomsen U, Hedley PL, Holm J. Leptin, Adiponectin and Their Ratio as Markers of Insulin Resistance and Cardiometabolic Risk in Childhood Obesity. Pediatr Diabetes (2020) 21 (2):194-202. doi: 10.1111/pedi.12964

53. Ren SM, Mei L, Huang H, Cao SF, Zhao RH, Zheng PY. Correlation Analysis of Gut Microbiota and Biochemical Indexes in Patients With Non-Alcoholic Fatty Liver Disease. Zhonghua Gan Zang Bing Za Zhi (2019) 27(5):369-75. doi: 10.3760/cma.j.issn.1007-3418.2019.05.009

54. Takada T, Kurakawa T, Tsuji H, Nomoto K. Fusicatenibacter Saccharivorans Gen. Nov., Sp. Nov., Isolated From Human Faeces. Int J Syst Evol Microbiol (2013) 63(Pt 10):3691-6. doi: 10.1099/ijs.0.045823-0

55. Song J, Qin Q, Li T, Ren F, Zhang H, Xie Y, et al. Impact of Carbohydrates on Autoinducer-2 Secretion of Bifidobacterium Longum Subsp. Longum BBMN68. Lett Appl Microbiol (2018) 66(4):340-6. doi: 10.1111/lam.12854

56. Zhu Y, Liu J, Lopez JM, Mills DA. Inulin Fermentation by Lactobacilli and Bifidobacteria From Dairy Calves. Appl Environ Microbiol (2020) 87(1): e01738-20. doi: 10.1128/AEM.01738-20

57. Xue J, Li X, Liu P, Li K, Sha L, Yang X, et al. Inulin and Metformin Ameliorate Polycystic Ovary Syndrome via Anti-Inflammation and Modulating Gut Microbiota in Mice. Endocr J (2019) 66(10):859-70. doi: 10.1507/ endocrj.EJ18-0567

58. Wang W, Zhai S, Xia Y, Wang H, Ruan D, Zhou T, et al. Ochratoxin A Induces Liver Inflammation: Involvement of Intestinal Microbiota. Microbiome (2019) 7(1):151. doi: 10.1186/s40168-019-0761-Z
59. Sun L, Xie C, Wang G, Wu Y, Wu Q, Wang X, et al. Gut Microbiota and Intestinal FXR Mediate the Clinical Benefits of Metformin. Nat Med (2018) 24 (12):1919-29. doi: 10.1038/s41591-018-0222-4

60. Qi X, Yun C, Sun L, Xia J, Wu Q, Wang Y, et al. Gut Microbiota-Bile AcidInterleukin-22 Axis Orchestrates Polycystic Ovary Syndrome. Nat Med (2019) 25(8):1225-33. doi: 10.1038/s41591-019-0509-0

61. Kang Y, Li Y, Du Y, Guo L, Chen M, Huang X, et al. Konjaku Flour Reduces Obesity in Mice by Modulating the Composition of the Gut Microbiota. Int J Obes (Lond) (2019) 43(8):1631-43. doi: 10.1038/s41366-018-0187-x

62. Natividad JM, Bruno L, Phuong PH, Marie-Laure M, Dominique R, Chantal B, et al. Bilophila Wadsworthia Aggravates High Fat Diet Induced Metabolic Dysfunctions in Mice. Nat Commun (2018) 9(1):2802-. doi: 10.1038/s41467018-05249-7

63. Lin H, An Y, Tang H, Wang Y. Alterations of Bile Acids and Gut Microbiota in Obesity Induced by High Fat Diet in Rat Model. J Agric Food Chem (2019) 67(13):3624-32. doi: 10.1021/acs.jafc.9b00249

Conflict of Interest: The authors declare that the research was conducted in the absence of any commercial or financial relationships that could be construed as a potential conflict of interest.

Publisher's Note: All claims expressed in this article are solely those of the authors and do not necessarily represent those of their affiliated organizations, or those of the publisher, the editors and the reviewers. Any product that may be evaluated in this article, or claim that may be made by its manufacturer, is not guaranteed or endorsed by the publisher.

Copyright (C) 2022 Wang, Xu, Liu, Wu, Gu, Zhang, Zhang, Fu, Ling, Wei, Luo, Shen, Zhao, Peng, Zhang and Ding. This is an open-access article distributed under the terms of the Creative Commons Attribution License (CC BY). The use, distribution or reproduction in other forums is permitted, provided the original author(s) and the copyright owner(s) are credited and that the original publication in this journal is cited, in accordance with accepted academic practice. No use, distribution or reproduction is permitted which does not comply with these terms. 